OBETS. Revista de Ciencias Sociales

Vol. 16, no 2, 2021, pp. 361-376

ISSN: 1989-1385

https://doi.org/10.14198/OBETS2021.16.2.09

\title{
INFODEMIA Y PERCEPCIÓN DEL RELATO MEDIÁTICO: LOS ESTUDIANTES DE PERIODISMO EN ESPAÑA ANTE LA COVID-19 ${ }^{1}$
}

\author{
INFODEMIC AND PERCEPTION OF MEDIA DISCOURSE: STUDENTS OF JOURNALISM \\ IN SPAIN FACE WITH THE COVID-19
}

\author{
Virginia Martín Jiménez \\ Universidad de Valladolid, España \\ virginia.martin@uva.es \\ https://orcid.org/0000-0002-7099-1060

\section{Itziar Reguero Sanz} \\ Universidad Complutense de Madrid, España \\ ireguero@ucm.es \\ https://orcid.org/0000-0002-3274-1861
}

\author{
Pablo Berdón Prieto \\ Universidad de Valladolid, España \\ pablo.berdon@uva.es \\ https://orcid.org/0000-0002-5279-4426 \\ Jacobo Herrero Izquierdo \\ Universidad de Valladolid, España \\ jacobo.herrero@uva.es \\ https://orcid.org/0000-0001-5672-3505
}

Cómo citar / Citation: Martín, V.; Reguero, I.; Berdón, P. y Herrero, J. (2021) "Infodemia y percepción del relato mediático: los estudiantes de periodismo en España ante la COVID-19”. OBETS. Revista de Ciencias Sociales, 16(2): 361-376. https://doi.org/10.14198/ OBETS2021.16.2.09

(C) 2021 Virginia Martín Jiménez, Itziar Reguero Sanz, Pablo Berdón Prieto, Jacobo Herrero Izquierdo

Este es un artículo de acceso abierto distribuido bajo los términos de la licencia de uso y distribución Creative Commons Reconocimiento 4.0 Internacional (CC BY 4.0) https://creativecommons.org/licenses/by/4.0/deed.es

Recibido: 20/09/20. Aceptado: 18/01/21

\begin{abstract}
Resumen
El objetivo principal de esta investigación es conocer la opinión acerca del "periodismo de pandemia» por parte del alumnado de Periodismo de las Universidades españolas. El método aplicado fue la encuesta de opinión, con una confianza muestral del 99,23\% y un error típico del 5\%. Los criterios de estratificación adoptados fueron las distintas universidades que imparten Periodismo y el número de estudiantes matriculados en cada sede. Los resultados revelan que los estudiantes son sensibles al fenómeno de la «infodemia», así como con la veracidad, y afirman haber sido víctimas de las noticias falsas.
\end{abstract}

Palabras clave: COVID-19; Infodemia; Periodismo; Universidad; Encuesta.

\begin{abstract}
This research project aims to know the opinion about the "pandemic journalism" on the part of the Journalism students of Spanish Universities. The method applied was the opinion survey, with 685 tests performed; which gives a sample confidence of $99,23 \%$ with a standard error of $5 \%$. The different universities that provide journalism studies and the number of students enrolled at each site were considered as the stratification criteria adopted. The results shows that students are largely critical of the phenomenon of "infodemic", as well as of the media accuracy and claim to be victims of fake news.
\end{abstract}

Keywords: COVID-19; Infodemic; Journalism; University; Survey.

1 Esta investigación es resultado del proyecto "Politainment en el entorno de la posverdad: nuevas narrativas, clickbait y gamificación" (Ref. CSO2017-84472-R), financiado por el Ministerio de Economía y Competitividad (Gobierno de España). 


\section{Extended abstract}

On 14 March 2020, the President of the Spanish Government, Pedro Sánchez, appeared on television after the meeting of the Council of Ministers to announce the declaration of the state of alarm as a result of the dizzying increase in the number of people affected by the disease known as COVID-19. The extraordinary situation, described as a public health emergency by the World Health Organisation (WHO) (WHO, 2020a), led firstly to the imposition of a series of restrictive measures and the beginning of a lockdown period which would last for the following three months. Secondly, and collaterally, the SARS-CoV-2 coronavirus pandemic brought about the creation of a new paradigm in which the classic forms of social relations were temporarily prohibited and where the continuous flow of data and information characterised that period.

In this context, the media played a decisive role, joining, together with other sectors such as healthcare, food, energy, banking or transport, the group of services that were understood from the beginning as essential. In this way, journalism continued with its usual pre-pandemic performance, although it was inevitably affected by the great confusion and general uncertainty that pervaded society. In addition to delivering its s traditional functions of informing, educating and entertaining, it was necessary to maintain its quality and informative rigour. All this with the added difficulty of the impact caused by a catastrophe of global dimensions on an extremely mediatized society.

Despite the recent appearance of COVID-19, its undeniable social, political and economic importance has spawned great deal of research work, also in the field of Communication and Social Sciences. The almost exclusive prominence of the virus in the media scene in recent months has encouraged the formulation of multiple hypotheses, questions and concerns that have in turn fuelled the reactivation of old debates related to the social responsibility of the media, their role within a democratic system of freedoms or the danger of fake news in the so-called information society, among others. In this context, the work presented here under the title "Infodemics and the perception of the media story: Journalism students at Spanish universities confront COVID-19" aims to collect and analyse the opinions of a specific group, Spanish journalism undergraduates, regarding the work of the media at the critical juncture posed by the pandemic.

With the advent of this "pandemic journalism", whose development has underlined the importance of good journalistic practice and correct media literacy on the part of the audience, and, at the same time, has warned of the danger of an overabundance of information in the post-truth era, a detailed analysis of the work of the mass media in this situation is a necessary task which this research intends to perform. By using the survey method, this paper focuses on a sociological profile with specific characteristics, as our target audience are special prosumers due to their training as future members of the journalistic profession. In fact, one of the novel contributions that this study seeks to make is related to its particular approach and the implemented methodology, which is based on surveying journalism students from across the country (with representation from all universities, both public and private) with a $99 \%$ confidence rate and a standard error of $5 \%$.

To carry out this task, a questionnaire was designed and divided into three sections according to the type of question asked: socio-demographic, on media consumption and on information processing. In total, 24 questions aimed at determining precisely not only the habits of information consumption, but also the assessment of the contents received, its quality and reliability or its potential errors, always from the critical eye expected from students of this discipline. The field work was completed between 25 May 2020 , coinciding with the entry of all the territories into one of the phases of de-escalation, and on 7 June of the same year, with the state of alarm still in force. It should be noted that the dissemination method combined several techniques, including communications by e-mail and through the various social media platforms (Twitter, Facebook, Telegram and LinkedIn).

The data extracted show the initial strength of television as the preferred medium for following the declaration of the state of alarm and its subsequent loss of prominence in favour of the digital press and the social networking services. A loss of confidence is also perceived as regards the mass media; whose recognition of essentiality did not translate into increased credibility on the part of respondents. The overabundance of information, the excessive ideologization of content or the vulnerability of students to the phenomenon of "infodemics" are issues which are also highlighted in the results. The analysis of the latter, moreover, was made taking into account variables such as the degree of affection for the disease, the class the students were enrolled in or their academic performance, in order to achieve more exhaustive results.

Given the nature of this research, its scope and its conclusions, this article aims to go one step further and add another point of view to the other contributions made to date about the informative impact of coronavirus 2019-nCoV. In short, it seeks to highlight the perspective on the media story entertained by a profile of respondents possessing specific traits and insights by their background and critical views. The coronavirus crisis has underlined the key role of the media as informers and public service, but at the same time it has revealed its possible shortcomings, problems and current difficulties. This study identifies some of them, and helps to determine the strengths and weaknesses of journalism at a truly critical time. 


\section{INTRODUCCIÓN: PLANTEAMIENTO DE LA INVESTIGACIÓN}

El 31 de diciembre de 2019, la Organización Mundial de la Salud (OMS) tuvo información de varios casos de neumonía de causa desconocida detectados en la ciudad de Wuhan, provincia de Hubei (China) (OMS, 2020a). A finales del mes de enero de 2020, la OMS definió la situación como de emergencia de salud pública de interés internacional por el impacto y por la difusión de la enfermedad bautizada como COVID-19 (OMS, 2020b), cuya expansión llevó a que dicha organización declarase, el 11 de marzo, pandemia al brote del nuevo coronavirus SARS-CoV-2 (OMS, 2020c).

En España, el 14 de marzo, tras el vertiginoso crecimiento de infectados, se aprueba el Real Decreto 463/2020 que da inicio a un estado de alarma de tres meses de duración que llevó al confinamiento en sus hogares de la ciudadanía hasta el comienzo de la «desescalada» el 4 de mayo (BOE, 2020). Durante ese período, profesionales de los sectores esenciales como la sanidad, la alimentación, la energía, el transporte, la banca o la comunicación mantuvieron su actividad laboral. Y prueba de ello es que el periodismo informativo continuó con el desempeño habitual anterior a la pandemia en un contexto global marcado por la transformación de los usos y costumbres tanto de emisores como de receptores (Masip, Aran-Ramspott, Ruiz-Caballero, Suau, Almenar y Puertas-Graell, 2020).

El impacto internacional de esta enfermedad en la conocida como sociedad de la información y el peso que han tomado ante este fenómeno los medios de comunicación (Casero-Ripollés, 2020; LázaroRodríguez; Herrera-Viedma, 2020), reforzando así su función social y democrática de informar y construir opinión (Habermas, 2006) junto con los debates planteados con anterioridad relacionados con la responsabilidad social de los medios, las fake news (Alonso, 2019), la libertad de prensa o la saturación informativa (conocida ya como «infodemia») (Kovach; Rosenstiel, 2007), conduce a estudiar la recepción del discurso sobre la COVID-19 en los jóvenes, y especialmente entre los estudiantes universitarios que se están formando para ejercer el periodismo en un futuro próximo.

Así, la aproximación a los estudiantes de Periodismo de las Universidades españolas y a su percepción de la "infodemia" -como término utilizado para referirse a la propagación de desinformación sobre la pandemia de la COVID-19, bien como producto de la sobreabundancia informativa o directamente por la circulación de noticias falsas (García Marín, 2020)- constituye el principal propósito de esta investigación. El perfil de prosumidor especial que representa este colectivo, así como su pertenencia a la sociedad mediatizada, hace especialmente interesante el examen de sus opiniones respecto al denominado "periodismo de pandemia". Para ello, las hipótesis y objetivos que se plantean son los siguientes:

H1.: Los estudiantes de periodismo valoraron en términos mayoritariamente negativos la labor informativa de los mass media durante la pandemia. Se parte de la premisa de que el periodismo actual atraviesa una cierta crisis de confianza, de ahí que el primer objetivo específico fuese conocer la imagen de la que gozaban, en términos de credibilidad, profesionalidad y estatus, los medios de comunicación.

H2.: Los estudiantes de periodismo optaron en gran medida por las plataformas digitales para informarse sobre la evolución de la pandemia y concedieron a los medios tradicionales (prensa, radio y televisión) un papel secundario. Un segundo objetivo de este trabajo era analizar las tendencias en el consumo de la información y conocer las vías más utilizadas para acceder a los contenidos mediáticos en un momento de emergencia que requería la actualización constante de datos. En ese sentido, las posibles respuestas contaban con un valor añadido, al proceder de los futuros integrantes de la profesión periodística.

H3.: Existió una correlación entre el grado de afección a la COVID-19 y el interés prestado al seguimiento mediático de la situación epidemiológica. Se pretendía conocer si los individuos que se vieron afectados por la enfermedad, tanto a nivel sanitario, económico o ambos, se comportaron de manera distinta en lo correspondiente al consumo de información y la valoración de la praxis mediática.

\section{ESTADO DE LA CUESTIÓN}

A pesar de la reciente aparición de la COVID-19, su destacado impacto social, político, económico y mediático ha generado la puesta en marcha de 
investigaciones que han recibido una rápida acogida por parte de las revistas académicas y por la comunidad científica ávida de conocimiento sobre la realidad que se está viviendo. Así lo demuestran los trabajos que se han centrado en estudios bibliométricos de la producción científica sobre el nuevo coronavirus (Alba-Ruiz, 2020; Chahrour, et al., 2020; Torres-Salinas, 2020).

En relación con la presente investigación, destacan los artículos sobre el coronavirus y los medios de comunicación (Lázaro-Rodríguez y Herrera-Viedma, 2020; Núñez Gómez et al., 2020; Herrero Izquierdo et al., 2021), mientras que otros papers han optado por analizar el efecto de la pandemia desde el consumo de los medios (Casero, 2020), de la comunicación corporativa (Xifra, 2020), de la manera en la que se ha informado desde las voces oficiales (Costa-Sánchez; López-García, 2020). No obstante, en lo que se ha hecho especial hincapié es en el fenómeno de las fake news (Checa y Olmos, 2020; Cifuentes Faura, 2020; García Marín, 2020; Gutiérrez Coba et al., 2020; López Borrull, 2020; Pérez Curiel y Velasco Molpeceres, 2020; Pérez Dasilva et al., 2020; Román San Miguel et al., 2020; Villa Gracia y Cerdán Martínez, 2020). Algunas de estas investigaciones, al igual que la presente, utilizan la encuesta como método para estudiar el fenómeno mediático en la era covid (López Rico et al., 2020; Montaña Blasco et al., 2020).

Desde el punto de vista de los hábitos de consumo mediático, se ha analizado la manera en la que esta nueva realidad global ha transformado los usos y costumbres tanto de emisores como receptores (Masip, Aran-Ramspott et al., 2020). Por otra parte, las redes sociales han despertado también interés entre la comunidad científica que ha optado por estudiar los contenidos sobre la COVID-19 difundidos en las diferentes plataformas digitales y el comportamiento de los usuarios (Thelwall; Thelwall, 2020; Thelwall; Levitt, 2020).

Partiendo de estos avances, este artículo busca dar un paso más y acercarse al impacto informativo del coronavirus 2019-nCoV, pero desde la percepción por parte de los estudiantes de periodismo de ese relato mediático; se trata de un colectivo de prosumidores con unos rasgos diferenciales a los de su grupo de pares, al estar recibiendo una formación especializada que les permite tener una mirada diferenciada como juez y parte frente a los medios y los contenidos que difunden.

\section{METODOLOGÍA}

De cara a la consecución de los objetivos, se planteó un tipo de investigación descriptiva en busca de examinar el fenómeno planteado y caracterizarlo del mejor modo posible (Hyman, 1971; De Vaus, 2001). Para ello, se diseñó una encuesta de opinión anónima autoadministrada por internet y dirigida a los 18.886 alumnos de Periodismo matriculados en las diferentes universidades de España, tanto de titularidad pública como privada, según los datos que recoge el Ministerio de Ciencia, Innovación y Universidades en su página web con fecha última de actualización del 18 de junio de 2019 (Ministerio de Educación, 2019). Esta población resulta muy interesante para realizar un estudio relacionado con los medios de comunicación porque sus miembros son un grupo prosumidor especial, está relacionado intensamente con el objeto de estudio, es más mensurable que otros grupos como los profesionales del periodismo o la población en general $y$, gracias a la alta oferta de estudios de periodismo en España, permite que el estudio tenga cierta pluralidad e impacto por todo el territorio, mejorando la representatividad a nivel estatal.

El cuestionario elaborado está dividido en tres sectores de acuerdo al tipo de pregunta realizada: sociodemográficas, sobre el uso mediático y sobre la valoración del tratamiento informativo. En total, la encuesta se compone de 24 preguntas con un tiempo medio de cumplimentación no superior a los 15 minutos, evitando así la saturación y/o el rechazo del sujeto encuestado (Padua et al, 1987).

En cuanto a la tipología de las preguntas, se trata de un cuestionario compuesto por preguntas cerradas con opción múltiple (19), y, en menor medida, por preguntas abiertas (5). La razón de incluir estas últimas, al margen de las que se utilizaron para conocer la edad del encuestado y hacer una mejor clasificación demoscópica, reside en el interés por profundizar en cuestiones relativas al consumo mediático. Es decir, para obtener una relación de los medios de comunicación más consultados por los individuos.

El método de distribución combinó varias técnicas. Por un lado, se optó por la difusión vía correo electrónico, a la que precedió un trabajo de búsqueda y recopilación de las direcciones de mail recogidas en las páginas web de las universidades españolas o en los 
planes docentes de los diferentes grados en Periodismo, para lograr la mayor difusión entre el alumnado. Por otro, se desplegó una campaña a través de diversas plataformas de redes sociales, en concreto: Twitter, Facebook, Telegram y LinkedIn. En el caso de Twitter y Facebook, la elección de estas plataformas estuvo motivada por su alta implantación en la sociedad. Telegram y LinkedIn se utilizaron porque tienen un perfil más profesional enfocado a grupos de trabajo concretos y, por tanto, permiten contactar de forma directa con la población de estudio. El trabajo de campo se realizó entre los días 25 de mayo de 2020, coincidiendo con la entrada de todos los territorios en alguna de las fases de la desescalada y, por tanto, dejando atrás la etapa más restrictiva del confinamiento, y el 7 de junio del mismo año. Puesto que el enfoque de la investigación pretende profundizar en particularidades del periodismo durante la pandemia, se consideró que las preguntas debían referirse a los momentos más extremos del periodo, es decir, el del confinamiento domiciliario. Se esperó, por tanto, al momento justo en el que ese periodo terminara en todos los territorios del país para que los encuestados partieran de momentos similares y que el paso del tiempo no desdibujara su opinión. Conviene señalar que, previo paso a la distribución de la encuesta, se realizó una prueba piloto o pretest encaminado a reducir las posibles deficiencias del cuestionario.

Para garantizar una mayor representatividad, se llevó a cabo un sistema de muestreo aleatorio y estratificado entre todos los alumnos de las distintas titulaciones en Periodismo de las universidades de España. Gracias a este procedimiento se aseguró que todas las instituciones que forman parte del universo de estudio participaran en la investigación (Gráfico 1). En la etapa de diseño muestral, se proyectó el estudio con una confianza del 99\% y un error típico del 5\%, sin embargo, para mejorar la representatividad y gracias a la alta participación del universo de estudio se aumentó al 99,23\% manteniendo el mismo porcentaje de error.

En total, se realizaron 685 encuestas distribuidas entre todas las universidades españolas tal y como muestra el Gráfico 1. Si se atiende al perfil de sociodemográfico de los encuestados, se observa una mayoría de respuestas procedentes de estudiantes residentes en zonas urbanas $(89,3 \%)$, por encima de los que viven en zonas intermedias $(6,4 \%)$ o rurales $(2,5 \%)$. También se percibe una mayor participación de los estudiantes matriculados en el último curso del Grado (Gráfico 2), así como un mayor número de mujeres $(66,1 \%)$ frente a hombres $(36,5 \%)$. Este dato coindice con la distribución que hace el propio Ministerio de Universidades, que divide el alumnado de esta carrera de la siguiente forma: 40,0878958\% alumnos y 59,9121042\% alumnas (Ministerio de Educación, 2019).

Gráfico 1. Alumnos por Comunidad Autónoma de procedencia de sus universidades.

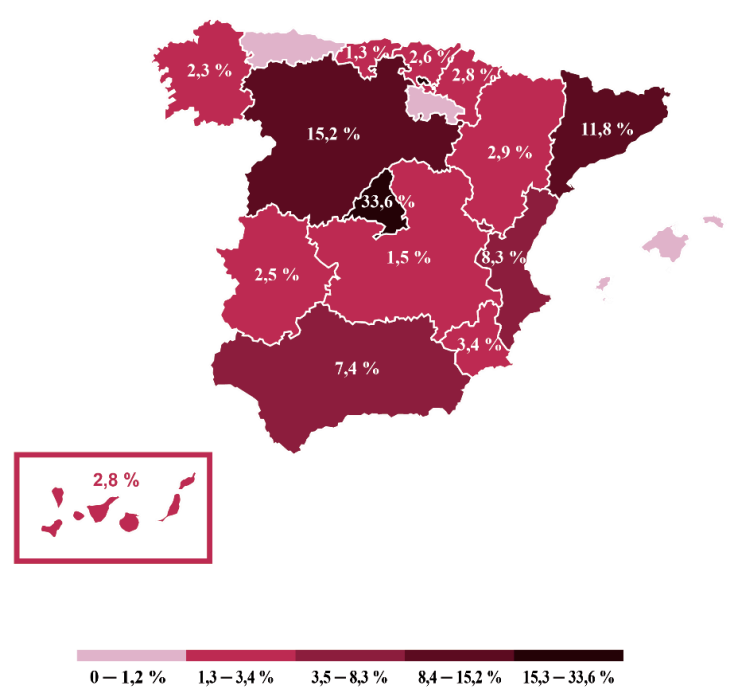

Fuente: Elaboración propia.

Gráfico 2. Curso académico 2019/2020.

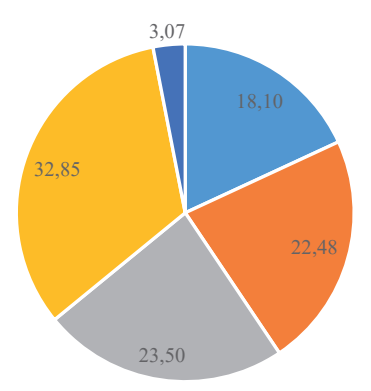

- Primero (\%) - - Segundo (\%) $\quad$ " Tercero (\%) $\quad$ "Cuarto (\%) $\quad$ - (en blanco) $(\%)$

Fuente: Elaboración propia. 


\section{RESULTADOS}

La declaración del estado de alarma en España se produjo el 13 de marzo 2020; un momento excepcional y de gran impacto mediático que fue seguido en directo por casi la mitad de los estudiantes encuestados (47,30\%) a través de la televisión. Un 14,31\% de los mismos, en cambio, siguió aquel histórico acontecimiento por redes sociales y un $6,57 \%$ lo hizo mediante prensa digital. En el contexto de una comparecencia muy esperada por la opinión pública, anunciada previamente, y con un país en situación de "preconfinamiento", como se puede ver en el Gráfico 3, la televisión acaparó buena parte de las miradas.

Gráfico 3. ¿Por qué vía se enteraron los estudiantes de la implantación del estado de alarma?

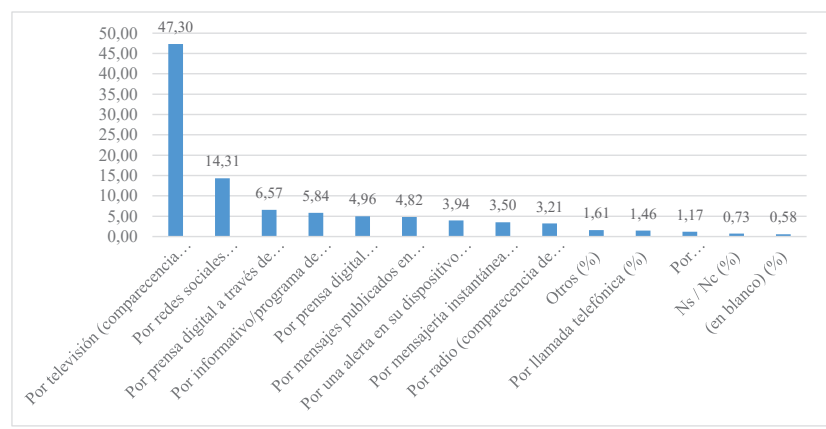

Fuente: Elaboración propia.

A partir de este instante, de este punto de inflexión que marcó el inicio de una nueva cronología, el consumo mediático de un $79,42 \%$ de los estudiantes fue in crescendo, mientras que únicamente en un $14,16 \%$ no tuvo variación y en un 5,69\% disminuyó. Ese aumento generalizado vino de la mano del establecimiento de unas pautas de consumo marcadas por lo que podríamos llamar unas "rutinas diarias de búsqueda de información"; así la mayoría de sujetos encuestados (34,31\%) buscó información sobre la COVID -19 entre dos y cuatro veces al día y solo un $11,24 \%$ se informó cinco veces o más en 24 horas. Además, los géneros informativos han sido los más consumidos (71,39\%), quedando en un segundo plano los interpretativos $(12,99 \%)$ y, en un tercero, los de opinión (4,38\%).

Según fueron mejorando los datos epidemiológicos, la tendencia ascendente en el consumo mediático solo se reflejó en un 23,36\% de los casos. El 29,64\% de los estudiantes se mantuvo constante y el 45,84\% rebajó su consumo de información de manera progresiva. Puede plantearse que ese descenso generalizado podría deberse a la saturación informativa recibida o a la necesidad de mantener cierta desconexión con la realidad vinculada a la COVID-19; sin embargo, cabe destacar que se observa que el sector del alumnado que mayoritariamente mantuvo invariable los niveles de consumo informativo a lo largo del confinamiento fue aquel que se sufrió las consecuencias de la existencia del virus afectándole, única y exclusivamente, a su salud $(38,33 \%)$.

Gráfico 4. Relación entre los distintos grados de afección de la COVID-19 con el consumo de información a lo largo del confinamiento.

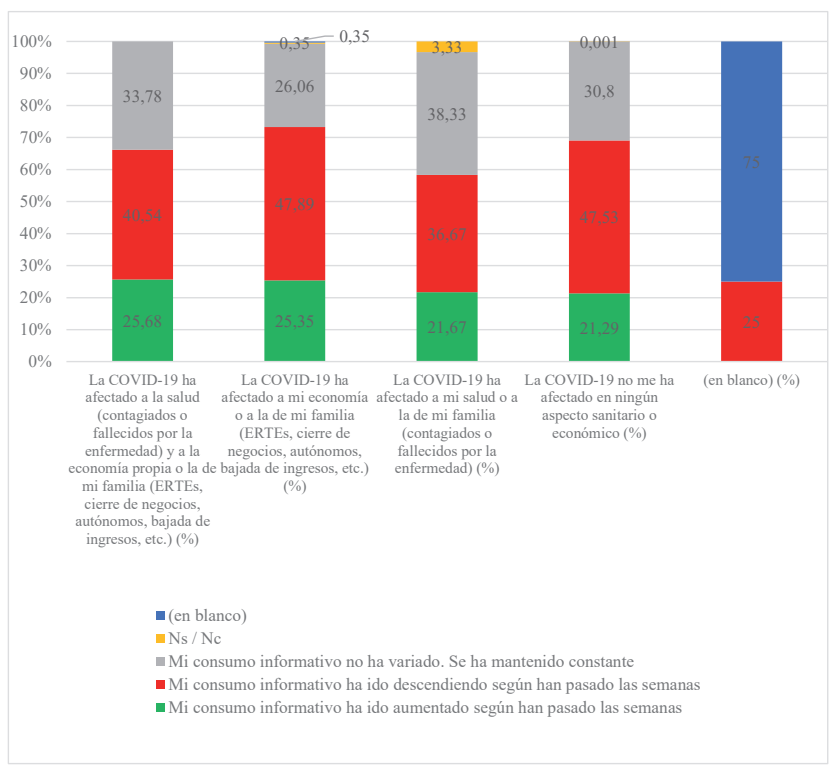

Fuente: Elaboración propia.

Pese a la centralidad televisiva inicial, la principal plataforma utilizada para acceder diariamente a la información fueron los diarios digitales $(44,09 \%)$, así como las redes sociales $(23,07 \%)$. De esta forma, una temprana conclusión que puede inferirse, y a la que se volverá más adelante, es que la pandemia ha confirmado dos tendencias en cuanto al consumo periodístico entre los más jóvenes que venían atisbándose desde hacía tiempo: el éxito creciente de los contenidos digitales -prensa y redes sociales- y la escasa popularidad de la prensa en papel $(1,02 \%)$.

Por otro lado, destacan los bajos porcentajes de usuarios que recurrieron para informarse a las comparecencias institucionales $(3,8 \%)$ y los servicios 
de mensajería instantánea, que solamente concentraron un $0,29 \%$ de la estadística. Este último dato puede atribuirse a la relación de plataformas como WhatsApp con el auge de las fake news en la era de la posverdad (Alonso, 2019), lo que explica que, como puede observarse en la Tabla 1, buena parte de la muestra descartase la utilización de esta herramienta para una útil y veraz consulta de la información.

Tabla 1. Las vías de información principales y secundarias para informarse sobre la COVID-19.

\begin{tabular}{|c|c|c|c|}
\hline Vía de información & Principal (\%) & Secundaria (\%) & Total (\%) \\
\hline Periódicos digitales & 44,09 & 22,34 & 33,21 \\
\hline $\begin{array}{l}\text { Redes sociales } \\
\text { (Facebook, Twitter, } \\
\text { Instagram, etc.) }\end{array}$ & 23,07 & 29,78 & 26,42 \\
\hline $\begin{array}{l}\text { Informativos de } \\
\text { televisión }\end{array}$ & 20,00 & 29,05 & 24,53 \\
\hline $\begin{array}{l}\text { Comparecencias } \\
\text { institucionales }\end{array}$ & 3,80 & 7,74 & 5,77 \\
\hline Radio & 3,50 & 4,82 & 4,16 \\
\hline $\begin{array}{l}\text { Otros programas } \\
\text { de televisión }\end{array}$ & 1,75 & 1,31 & 1,53 \\
\hline $\begin{array}{l}\text { Boletín Oficial del } \\
\text { Estado (BOE) }\end{array}$ & 1,46 & 0,73 & 1,09 \\
\hline Periódicos impresos & 1,02 & 1,31 & 1,17 \\
\hline $\begin{array}{l}\text { Servicios de } \\
\text { mensajería } \\
\text { (WhatsApp, } \\
\text { Telegram, etc.) }\end{array}$ & 0,29 & 1,31 & 0,80 \\
\hline (En blanco) & 0,58 & 0,88 & 0,73 \\
\hline $\mathrm{Ns} / \mathrm{Nc}$ & 0,44 & 0,73 & 0,58 \\
\hline
\end{tabular}

Fuente: Elaboración propia.

En este clima de importancia digital, la red social Twitter (255) fue la plataforma más utilizada por los estudiantes para informarse acerca de la pandemia, seguida El País (181) y de RTVE (116). Destaca a su vez el lugar que ocupa Instagram en esta escala, pues se sitúa en número de usuarios (22) por encima de Facebook. Asimismo, cabe indicar el notable protagonismo de TV3 y La Vanguardia como medios de ámbito autonómico, ambos adscritos a Cataluña.
Gráfico 5. Las quince empresas / plataformas preferidas para informarse sobre la COVID-19.

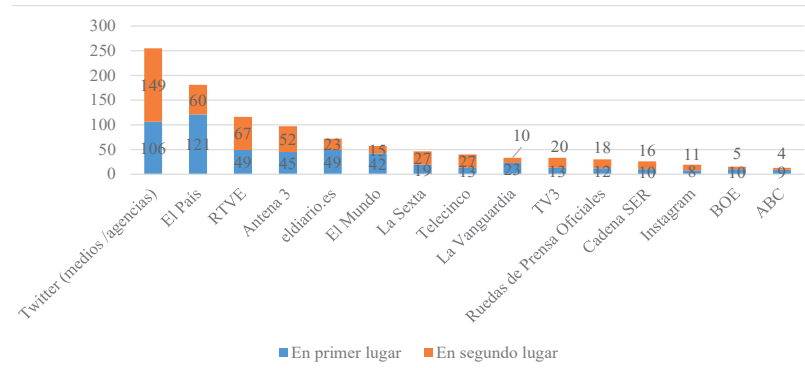

Fuente: Elaboración propia.

El elevado consumo informativo, sin embargo, contrasta con la escasa confianza otorgada a los medios de comunicación. Para un 65,55\%, la llegada de la pandemia no supuso un aumento de la credibilidad de los mass media y solo un $26,28 \%$ expuso un pensamiento contrario. Como dato relevante, puede destacarse un mayor posicionamiento crítico respecto a esa confiabilidad en la labor periodística por parte del alumnado con mayor formación. Así, un 70,22\% de los estudiantes de cuarto niega que la pandemia derivase en un aumento de la credibilidad de los medios, seguido de un $68,94 \%$ en tercero, de un $63,71 \%$ en primero y de un $58,44 \%$ en segundo. En este sentido, también se establecen diferencias con base en si el alumnado realizó (o no) prácticas profesionales durante el estado de alarma. Pese a que, en ambos casos, las cifras indican que los medios no ganaron confianza, el porcentaje es mayor cuando no trabajaron $(67,54 \%)$ que cuando sí lo hicieron $(57,41 \%)$, lo cual revela que la proximidad al ejercicio profesional rebajó los juicios negativos sobre el tratamiento informativo.

De forma paralela, un 48,91\% de los encuestados considera que ha habido una excesiva información en torno a la COVID-19, aunque deben puntualizase dos cuestiones interesantes cuyos datos pueden consultarse en las tablas que se insertan a continuación. La primera demuestra que cuanto menos ha afectado el virus a los estudiantes (tanto a nivel económico como sanitario), menos interés han mostrado por los contenidos mediáticos referidos al mismo. La segunda, que los encuestados que han realizado prácticas externas y han tenido sus primeros contactos con el desempeño de la labor periodística consideran en una mayor proporción que el volumen de información ha sido el preciso, teniendo en cuenta la situación que se estaba viviendo en el país. 
Tabla 2. Relación entre el tratamiento cuantitativo de la información por los medios con el grado de afección por la COVID-19.

\begin{tabular}{|c|c|c|c|c|c|}
\hline \multirow[b]{2}{*}{ Abundancia informativa } & \multicolumn{5}{|c|}{ Grado de afección del COVID-19 (\%) } \\
\hline & $\begin{array}{l}\text { La COVID-19 ha afectado } \\
\text { a la salud (contagiados } \\
\text { o fallecidos por la } \\
\text { enfermedad) y a la economía } \\
\text { propia o la de mi familia } \\
\text { (ERTEs, cierre de negocios, } \\
\text { autónomos, bajada de } \\
\text { ingresos, etc.) (\%) }\end{array}$ & $\begin{array}{l}\text { La COVID-19 } \\
\text { ha afectado a mi } \\
\text { economía o a la de } \\
\text { mi familia (ERTEs, } \\
\text { cierre de negocios, } \\
\text { autónomos, bajada de } \\
\text { ingresos, etc.) (\%) }\end{array}$ & $\begin{array}{l}\text { La COVID-19 ha } \\
\text { afectado a mi salud } \\
\text { o a la de mi familia } \\
\text { (contagiados o } \\
\text { fallecidos por la } \\
\text { enfermedad) (\%) }\end{array}$ & $\begin{array}{l}\text { La COVID-19 } \\
\text { no me ha } \\
\text { afectado en } \\
\text { ningún aspecto } \\
\text { sanitario o } \\
\text { económico (\%) }\end{array}$ & (en blanco) (\%) \\
\hline $\begin{array}{l}\text { En general, los medios } \\
\text { están ofreciendo } \\
\text { excesiva información } \\
\text { sobre la COVID-19 }\end{array}$ & 45,95 & 45,77 & 48,33 & 53,61 & 25,00 \\
\hline $\begin{array}{l}\text { En general, los medios } \\
\text { están ofreciendo poca } \\
\text { información sobre } \\
\text { la COVID-19 }\end{array}$ & 10,81 & 7,39 & 6,67 & 4,18 & 0,00 \\
\hline $\begin{array}{l}\text { En general, los medios } \\
\text { están ofreciendo una } \\
\text { información proporcional } \\
\text { la relevancia social } \\
\text { de la COVID-19 }\end{array}$ & 39,19 & 44,72 & 43,33 & 39,92 & 0,00 \\
\hline $\mathrm{Ns} / \mathrm{Nc}$ & 4,05 & 1,76 & 1,67 & 2,28 & 0,00 \\
\hline (En blanco) & 0,00 & 0,35 & 0,00 & 0,00 & 75,00 \\
\hline
\end{tabular}

Fuente: Elaboración propia.

Tabla 3. Relación entre el tratamiento cuantitativo de la información por los medios con el desempeño de actividad profesional.

\begin{tabular}{|c|c|c|c|}
\hline & \multicolumn{3}{|c|}{ Desempeño de actividad profesional durante el Estado de Alarma (\%) } \\
\hline Tratamiento cuantitativo de la información & No $(\%)$ & Sí (\%) & (en blanco) (\%) \\
\hline $\begin{array}{l}\text { En general, los medios están ofreciendo } \\
\text { excesiva información sobre la COVID-19 }\end{array}$ & 51,23 & 37,96 & 50,00 \\
\hline $\begin{array}{l}\text { En general, los medios están ofreciendo } \\
\text { poca información sobre la COVID-19 }\end{array}$ & 6,67 & 5,56 & 0,00 \\
\hline $\begin{array}{l}\text { En general, los medios están ofreciendo } \\
\text { una información proporcional la } \\
\text { relevancia social de la COVID-19 }\end{array}$ & 40,00 & 53,70 & 25,00 \\
\hline $\mathrm{Ns} / \mathrm{Nc}$ & 2,11 & 2,78 & 0,00 \\
\hline (En blanco) & 0,00 & 0,00 & 100,00 \\
\hline
\end{tabular}

Fuente: Elaboración propia.

Por otra parte, un elevado porcentaje de los estudiantes (más del 75\%) reconocieron haber recibido fake news relacionadas con el coronavirus, las cuales creyeron ciertas en un primer instante. En cuanto a esto último, los alumnos matriculados en cursos superiores fueron más conscientes de haber recibido estos bulos (77,33\%), ya que el porcentaje decrece a medida que desciende el nivel formativo: un $77,02 \%$ para los matriculados en el tercero curso del Grado, un $72,73 \%$ para los de segundo y un $72,58 \%$ en primero.
Esa misma tendencia se ha observado con base en otra cuestión que se considera de especial relevancia. Un 37,66\% de los encuestados apunta que la mayoría de las informaciones que recibieron por parte de los medios de comunicación estaban sesgadas ideológicamente, porcentaje que, de nuevo, aumenta según avanzan los cursos: en primero, 30,65\%; en segundo, 35,71\%; en tercero, 39,13\%; y en cuarto, $41,33 \%$. De modo similar, la idea del sesgo ideológico de la información la corroboran en mayor medida 
aquellos que desempeñaron prácticas curriculares: un $44,44 \%$, frente a un $36,84 \%$.

Durante el estado de alarma se ha advertido que los contenidos mediáticos relativos a la COVID-19 estuvieron muy polarizados, posicionándose en contra o a favor de las medidas gubernamentales para sofocar la crisis, continuando una tendencia iniciada en los últimos tiempos (Cardenal et al., 2018). En consecuencia, los estudiantes aseguraron no estar viendo reflejados en el panorama mediático los principios periodísticos que se imparten en las aulas de la titulación. Las cifras son esclarecedoras: solo un $15,62 \%$ considera que la mayoría de las informaciones están perfectamente contrastadas y son veraces.

Gráfico 6. Resultados sobre la calidad de las informaciones proporcionadas por los medios.
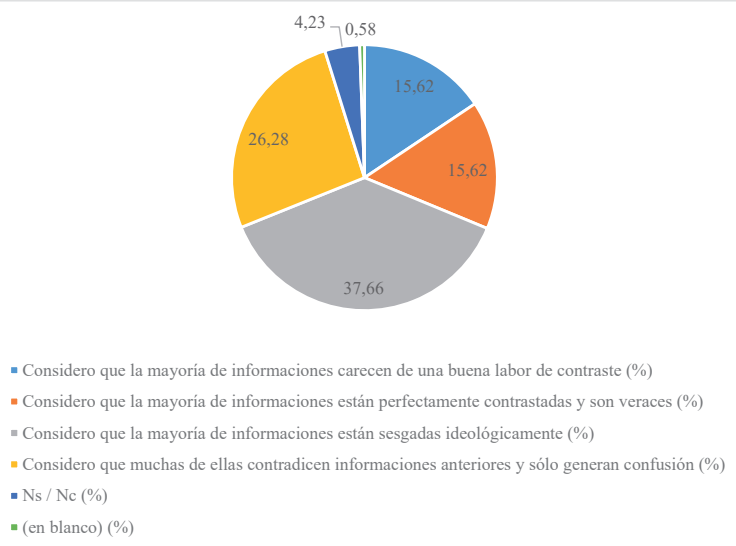

Fuente: Elaboración propia.

Al interrogante sobre qué tipo de contenido deben priorizar los medios informativos en una situación de crisis como la ocurrida, las respuestas se inclinan, en primer lugar, por las cuestiones sanitarias y las estadísticas diarias $(55,91 \%)$ y, en segundo término, por los contenidos formativos relacionados con la utilización de las mascarillas, el lavado de manos y otras cuestiones de higiene relacionadas con la "nueva normalidad" (27,15\%). Los menores porcentajes se encuentran en las opciones por las que fueron decantándose los medios a medida que se atravesaron los peores momentos de la pandemia: iniciativas solidarias $(1,9 \%)$ e historias de superación personal $(1,46 \%)$.
Gráfico 7. El tipo de información que deberían haber priorizado los medios en primer y segundo lugar.

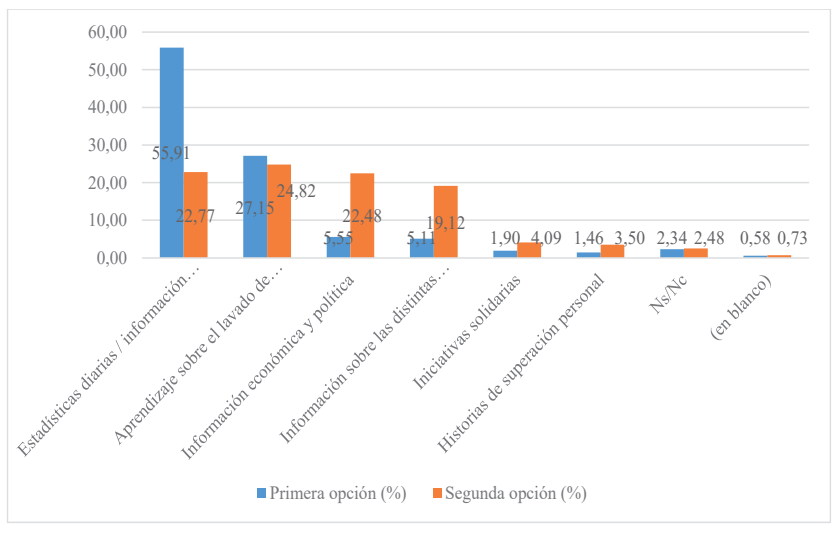

Fuente: Elaboración propia.

\section{CONCLUSIONES}

Las principales conclusiones que se extraen de este trabajo han permitido dar respuesta a una serie de interrogantes que se dieron al inicio de la pandemia de la COVID-19 y durante el desarrollo de la misma acerca de la labor de los medios de comunicación. Saber ya no solo cómo se informó el público sobre la crisis sanitaria, sino también la manera en la que fue percibida esa información ofrece una interesante panorámica que, en este caso, se forma mediante las respuestas de un colectivo especialmente implicado con el trabajo de los mass media, su responsabilidad social y su deber para con la ciudadanía. Uno de esos interrogantes, que además enlaza con la primera hipótesis de investigación, se concreta fundamentalmente en la siguiente pregunta: ¿cómo valoraron los estudiantes de periodismo el desempeño de los medios durante la situación provocada por el coronavirus?

Los estudios previos centrados en el impacto del coronavirus en el sistema mediático (Salaverría et al., 2020; Pérez Curiel y Velasco Molpeceres, 2020) ya advertían del alto desorden informativo provocado por la pandemia en el que era posible el desarrollo de un caldo de cultivo de imprecisiones, falacias y Noticas falsas. Sin embargo, este trabajo ha puesto de manifiesto cómo el fenómeno de la "infodemia" ha traído consigo una serie consecuencias que no afectan exclusivamente al usuario, al receptor, al consumidor de contenidos, sino también a los informadores. El elevado número de respuestas críticas en relación al desempeño de los mass media, así como la prevalencia de una sensación de 
sobreabundancia informativa, subraya la preocupante tesitura en la que se encuentra el periodismo actual. Dentro de los problemas derivados de la denominada sociedad mediática, destaca por encima del resto la evidente pérdida de confianza en los medios, la cual se ha hecho visible (y posiblemente haya aumentado) a lo largo de esta crisis. Los resultados obtenidos demuestran que la credibilidad del periodismo ha quedado en entredicho en esta etapa y ha servido para reforzar la imagen negativa de sus responsables frente a lo que correspondería ese atributo de "esencial" que se les concedió.

En relación con esto último, se han percibido dos realidades que podrían considerarse como causantes directos de la difícil situación que afrontan los medios de comunicación. En primer lugar, el fenómeno consolidado de los bulos o las fake news, que afectó, y de manera evidente, a este grupo de estudiantes, a priori, más preparado que el resto a la hora de contrarrestar sus efectos negativos. En ese sentido, cabe señalar la vulnerabilidad del alumnado de periodismo para combatir los contenidos falsos que circulan por la red. Los escasos índices de alfabetización mediática, junto a la preferencia de los encuestados por las plataformas digitales, potencia los riesgos de esta "infodemia" y evidencia las deficiencias en el aprendizaje de mecanismos y herramientas para hacer frente a las informaciones falsas. En segundo término, cabría señalar la percepción sobre una alta ideologización/ polarización de los contenidos publicados, lo cual no deja de ir en consonancia con el propio clima de confrontación política predominante en España. Se trata sin duda de otro síntoma preocupante que afectaría a la veracidad de las informaciones periodísticas, señalado anteriormente en estudios sobre la materia (Teruel Rodríguez, 2016).

Por otro lado, en lo que afecta al consumo mediático, ya se ha puesto de relieve el peso incontestable de las plataformas digitales (prensa diaria y redes sociales fundamentalmente) por encima de los medios tradicionales como la prensa en papel, la radio e incluso la televisión. Esta última sí logró ser el medio más seguido justo en el momento de la declaración oficial del estado de alarma, pero pronto cedió posiciones, quizá más de lo que se esperaba en un inicio. No deja de percibirse aquí un acontecimiento que recuerda a los estudios relacionados con los eventos mediáticos (media-events) y que destaca la habilidad de la televisión para hacer "historia en directo" permitiendo a los usuarios experimentar los hechos a través del escenario catódico y logrando imponer una atención universal (Dayan y Katz, 1995). Aun así, los resultados permiten confirmar la segunda hipótesis de esta investigación y reafirma una tendencia que ya venía consolidándose en los últimos años: el triunfo de lo digital y la cada vez menor atención a los viejos formatos.

En lo que respecta a la relación entre el grado de afección a la COVID-19 y el nivel de seguimiento informativo, el repentino aumento de la atención mediática a raíz del anuncio del confinamiento se redujo según fueron mejorando los datos epidemiológicos. Existió pues un estancamiento en las "rutinas diarias de búsqueda de información", y, posteriormente, un descenso de las mismas, aunque conviene hacer algunas apreciaciones. La mayoría de individuos que mantuvo invariables sus niveles de consumo informativo fueron aquellos que se vieron afectados por el virus exclusivamente a nivel de salud (tanto la suya personal como la de sus allegados). De igual modo, las personas menos perjudicadas por la enfermedad manifestaron estar recibiendo excesiva información sobre la pandemia, lo que permite deducir una correlación entre la situación personal y el comportamiento y la percepción del encuestado. Los matices, no obstante, son amplios, puesto que las consecuencias de la COVID-19 a nivel económico no evitaron el descenso de la atención informativa fruto también de esa sensación de sobreabundancia que se dio en todos los casos, con independencia de la cercanía o distancia con la que se encaró el SARS-CoV-2.

Por último, conviene realizar una serie de consideraciones en cuanto a la valoración de los estudiantes acerca del tipo de contenidos (géneros periodísticos) que proporcionaron los medios de comunicación. En un contexto en que la información fue bastante más consumida que los géneros interpretativos y la opinión, la demanda de las llamadas hard news (estadísticas de contagios, información sanitaria, política y economía, etc.) podría responder a la necesidad de obtener noticias, datos precisos y actualizados sobre la pandemia. También a la capacidad de los propios usuarios para acceder a los contenidos de entretenimiento/dispersión/soft news a través de otras vías o plataformas. Respondería a su vez a una 
exigencia de mayor rigor y seriedad a los medios de comunicación por parte de los estudiantes, o simplemente, como han argumentado algunos autores, al hecho de que las noticias, la información más pura, se ha convertido en un valiosísimo recurso para los ciudadanos en esta situación de emergencia (CaseroRipollés, 2020).

Así, y respondida la pregunta inicial sobre cómo valoraron los estudiantes de periodismo el desempeño de los mass media, cabría ahora trasladar el debate a la sociedad, acercar las presentes conclusiones a los profesionales de los medios y comparar su visión con la recogida en estas páginas. Los síntomas de las flaquezas del periodismo requieren la puesta en marcha de medidas precisas, como bien lo han señalado las generaciones que integrarán esta profesión en los años venideros. Cuestiones como ipor qué los estudiantes también se han visto afectados por las amenazas de las fake-news? ¿cómo combatir la desinformación? o ¿qué futuro depara a la prensa, radio y televisión ante el auge vertiginoso de las redes sociales como nuevos canales (medios) de información? abren múltiples y sustanciales vías de estudio. Los párrafos anteriores pueden ser un buen punto de partida.

\section{REFERENCIAS BIBLIOGRÁFICAS}

Alonso, M. (2019). "Fake News: desinformación en la era de la sociedad de la información". Ámbitos. Revista Internacional de Comunicación, (45) 29-52. https://doi. org/10.12795/Ambitos.2019.i45.03

Boletín Oficial del Estado (BOE), núm. 123, de 3 de mayo de 2020, pp. 31113-31117. https://www.boe.es/buscar/doc. php?id=BOE-A-2020-4792

Cardenal, A. S.; Galais, C.; Moré, J.; Cristancho, C.; MajóVázquez, S. (2018). "El reto de medir el sesgo ideológico en los medios escritos digitales". Quaderns del CAC, 31(44), 37-46. https://www.cac.cat/sites/default/ files/2018-08/Q44_ES.pdf

Casero-Ripollés, A. (2020). "Impact of Covid-19 on the media system. Communicative and democratic consequences of news consumption during the outbreak". El profesional de la información, 29(2). https://doi.org/10.3145/epi.2020. mar. 23

Checa y Olmos, F. (2020). "Miedo y comunicación en tiempo de pandemia: de las fake news al humor". En C. Vázquez Domínguez; B. Pérez González y D. Salzano. Cambio y coronavirus. Representaciones sociales: burla, silencio y miedo (pp. 29-44). Sevilla: McGraw Hill.

Cifuentes Faura, J. (2020). "Fake news during COVID-19: how to detect them?". Comunicación, (42), 100-103. http://dx.doi.org/10.18566/comunica.n42.a07
Costa-Sánchez, C.; López-García, X. (2020). "Comunicación y crisis del coronavirus en España. Primeras lecciones". El profesional de la información, 29(3). https://doi. org/10.3145/epi.2020.may.04

Chahrour, M.; Assi, S.; Bejjani, M.; Nasrallah, A. A.; Salhab, H.; Fares, M. Y.; Khachfe, H. H. (2020). "A bibliometric analysis of Covid-19 research activity: A call for increased output". Cureus, 12(3). https://doi.org/10.7759/ cureus. 7357

Dayan, D. y Katz, E. (1995). La Historia en Directo: La Retransmisión Televisiva de Los Acontecimientos. Gustavo Gil S.A Ediciones.

De Vaus, D. (2001). Research Design in Social Research. Londres: SAGE Publications Ltd.

García-Marín, D. (2020). "Infodemia global. Desórdenes informativos, narrativas fake y fact-checking en la crisis de la Covid-19". El profesional de la información, 29, (4). https://doi.org/10.3145/epi.2020.jul.11

Gutiérrez-Coba, L.; Coba-Gutiérrez, P., y Gómez-Díaz, J. (2020). "Noticias falsas y desinformación sobre el Covid-19: análisis comparativo de seis países iberoamericanos". Revista Latina de Comunicación Social, (78), 237-264. https://www.doi.org/10.4185/RLCS2020-1476

Hyman, H. (1971). Diseño y análisis de las encuestas sociales. Buenos Aires: Amorrotu Ediciones.

Habermas, J. (2006). "Political communication in media society: Does democracy still enjoy an epistemic dimension? The impact of normative theory on empirical research". Communication theory, 16(4), 411-426. https:// doi.org/10.1111/j.1468-2885.2006.00280.x

Herrero Izquierdo, J., Berdón Prieto, P., Reguero Sanz, I. y Martín Jiménez, V. (2021). "El discurso de El País y El Mundo ante la COVID-19". ComHumanitas: Revista Científica De Comunicación, 11(3), 23-40. https://doi. org/10.31207/rch.v1li3.265

Kovach, B.; Rosenstiel, T. (2007). The elements of journalism: What newspeople should know and the public should expect. New York: Three Rivers Press.

Lázaro-Rodríguez, P.; Herrera-Viedma, E. (2020). "Noticias sobre Covid-19 y 2019-nCoV en medios de comunicación de España: el papel de los medios digitales en tiempos de confinamiento". El profesional de la información, 29(3). https://doi.org/10.3145/epi.2020.may.02

López-Borrull, A. (2020). "Fake news e infodemia científica durante la Covid-19, ¿dos caras de la misma crisis informacional?". Anuario ThinkEPI, 14. https://doi. org/10.3145/thinkepi.2020.e14e07

López-Rico, C.; González-Esteban, J.L. y HernándezMartínez, A. (2020). "Polarización y confianza en los medios españoles durante el Covid-19. Identificación de perfiles de audiencia". Revista Española de Comunicación en Salud, suplemento, 1, 77 - 89. https://doi.org/10.20318/ recs.2020.5439

Masip, P.; Aran-Ramspott, S.; Ruiz-Caballero, C.; Suau, J.; Almenar, E.; Puertas-Graell, D. (2020). "Consumo informativo y cobertura mediática durante el confinamiento por el Covid-19: sobreinformación, 
sesgo ideológico y sensacionalismo". El profesional de la información, 29(3). https://doi.org/10.3145/epi.2020. may. 12

Ministerio de Ciencia, Innovación y Universidades. (2019). Sistema Integrado de Información Universitaria (SIIU). Estadísticas de Educación EDUCAbase. Secretaría General de Universidades. Recuperado de http:// estadisticas.mecd.gob.es/EducaDynPx/educabase/ index.htm? type=pcaxis \&path=/Universitaria/ Alumnado/1GradoCiclo/Matriculados/\&file=pcaxis\#

Montaña Blasco, M.; Ollé Castellà, C., y Lavilla Raso, M. (2020). "Impacto de la pandemia de Covid-19 en el consumo de medios en España". Revista Latina de Comunicación Social, (78), 155-167. https://www.doi. org/10.4185/RLCS-2020-1472

Núñez-Gómez, P.; Abuín-Vences, N.; Sierra-Sánchez, J. y Mañas-Viniegra, L. (2020). "El enfoque de la prensa española durante la crisis del Covid-19. Un análisis del framing a través de las portadas de los principales diarios de tirada nacional". Revista Latina de Comunicación Social, (78), 41-63. https://www.doi.org/10.4185/RLCS-20201468

OMS (2020a). Neumonía de causa desconocida - China. https:// www.who.int/csr/don/05-january-2020-pneumonia-ofunkown-cause-china/es/

OMS (2020b). 2019-nCoV outbreak is an emergency of international concern. World Health Organization. https:// www.euro.who.int/en/health-topics/health-emergencies/ coronavirus-covid-19/news/news/2020/01/2019ncovoutbreak-\%20is-an-emergency-of-internationalconcern

OMS (2020c). Alocución de apertura del Director General de la OMS en la rueda de prensa sobre la Covid-19 celebrada el 11 de marzo de 2020. World Health Organization. https://www. who.int/es/dg/speeches/detail/who-director-general-sopening-remarks-at-the-media-briefing-on-covid-19--11-march-2020

Padua, J.; Ahman, I.; Apezechea, H.; Borsotti C. (1987). Técnicas de investigación aplicadas a las ciencias sociales. Madrid: Esfera de los libros.

Pérez-Curiel, C. y Velasco Molpeceres, A. M. (2020). Impacto del discurso político en la difusión de bulos sobre Covid-19. Influencia de la desinformación en públicos y medios. Revista Latina de Comunicación Social, (78), 65-97. https://www.doi.org/10.4185/RLCS-2020-1469

Pérez-Dasilva, J.Á., Meso-Ayerdi, K. \& MendigurenGaldospín, T. (2020). "Fake news y coronavirus: detección de los principales actores y tendencias a través del análisis de las conversaciones en Twitter". Profesional De La Información, 29(3). https://doi.org/10.3145/ epi.2020.may.08

Román-San-Miguel, A.; Sánchez-Gey Valenzuela, N., y ElíasZambrano, R. (2020). "Las fake news durante el Estado de Alarma por COVID-19. Análisis desde el punto de vista político en la prensa española". Revista Latina de Comunicación Social, (78), 359-391. https://www.doi. org/10.4185/RLCS-2020-1481
Salaverría, R.; Buslón, N.; López-Pan, F.; León, B.; LópezGoñi, I.; Erviti, M. C. (2020). Desinformación en tiempos de pandemia: tipología de los bulos sobre la Covid-19. El profesional de la información, 29(3). https:// doi.org/10.3145/epi.2020.may.15

Teruel Rodríguez, L. (2016). "El impacto de la crisis política y económica sobre el impacto de los medios españoles". Historia y Comunicación Social 21(1), 203-220. https://doi. org/10.5209/rev_HICS.2016.v21.n1.52692

Thelwall, M.; Thelwall, S. (2020). "Covid-19 tweeting in English: Gender differences". El profesional de la información, 29(3). https://doi.org/10.3145/epi.2020. may.01

Thelwall, M.; Levitt, J. M. (2020). "Retweeting Covid-19 disability issues: Risks, support and outrage". El profesional de la información, 29(2). https://doi.org/10.3145/epi.2020. mar. 16

Torres-Salinas, D. (2020). "Ritmo de crecimiento diario de la producción científica sobre Covid-19. Análisis en bases de datos y repositorios en acceso abierto". El profesional de la información, 29(2). https://doi.org/10.3145/epi.2020. mar. 15

Villa Gracía, D., y Cerdán Martínez, V. (2020). "Bulos durante la pandemia del Covid-19 en España: un estudio a través de Google Trends". Revista Latina de Comunicación Social, (78), 169-182. https://www.doi.org/10.4185/ RLCS-2020-1473

Xifra, J. (2020). "Comunicación corporativa, relaciones públicas y gestión del riesgo reputacional en tiempos del Covid-19". El profesional de la información, 29(2). https:// doi.org/10.3145/epi.2020.mar.20

\section{NOTAS BIOGRÁFICAS}

\section{VIRGINIA MARTÍN JIMÉNEZ}

Profesora Titular del Área de Periodismo de la Universidad de Valladolid (UVa. España) y forma parte del Grupo de Investigación en Nuevas Tendencias en Comunicación (NUTECO). Ha participado en casi una decena de proyectos de investigación competitivos y actualmente es parte del equipo de "Politainment en el entorno de la posverdad: nuevas narrativas, clickbait y gamificación" (Ref. CSO2017-84472-R).

\section{ITZIAR REGUERO SANZ}

Profesora en la Facultad de Ciencias de Información de la Universidad Complutense de Madrid (UCM). (España). Doctora con mención internacional (Reino Unido), licenciada en Periodismo y Premio Extraordinario en el Máster en Investigación de 
la Comunicación como Agente Histórico Social (UVa). Sus líneas de investigación se centran en las relaciones de poder que se establecen entre políticos y medios, así como su posterior influencia en la sociedad.

\section{PABLO BERDÓN PRIETO}

Investigador predoctoral de la Universidad de Valladolid (UVa. España). Está Graduado en Publicidad y Relaciones Públicas y cuenta con un Máster en Investigación de la Comunicación como agente histórico-social (UVa). Sus estudios se enmarcan en la relación histórica de los medios de comunicación con el poder político.

\section{JACOBO HERRERO IZQUIERDO}

Investigador predoctoral en la Universidad de Valladolid (UVa. España). Graduado en Periodismo con Máster en Investigación de la Comunicación como Agente Histórico Social (UVa). Sus líneas de investigación se centran en el estudio del uso político de los medios de comunicación en momentos críticos de la historia. 


\section{ANEXO I \\ UN ANÁLISIS CRÍTICO DEL PAPEL DEL PERIODISMO DURANTE LA CRISIS DE LA COVID-19}

Primera parte de la encuesta: preguntas sociodemográficas

1.- Sexo

- Mujer

- Hombre

- Otro

- Prefiero no decirlo

2.- Edad

(Respuesta abierta)

3.- En qué universidad estudia el grado en periodismo:

- Universidad a Distancia de Madrid

- Universidad Abat Oliba CEU

- Universidad Antonio de Nebrija

- Universidad Autónoma de Barcelona

- Universidad Camilo José Cela

- Universidad Cardenal Herrera-CEU

- Universidad Carlos III de Madrid

- Universidad de Castilla-La Mancha

- Universidad Católica San Antonio de Murcia

- Universidad Complutense de Madrid

- Universidad Europea de Madrid

- Universidad Europea del Atlántico

- Universidad Europea Miguel de Cervantes

- Universidad de Extremadura

- Universidad Fernando Pessoa-Canarias

- Universidad Francisco de Vitoria

- Universidad de Girona

- Universidad de Illes Balears

- Universidad Internacional de Catalunya

- Universidad Jaume I de Castellón

- Universidad de La Laguna

- Universidad de Málaga

- Universidad Miguel Hernández de Elche

- Universidad de Murcia

- Universidad de Navarra

- Universidad del País Vasco/Euskal Herriko Unibertsitatea

- Universidad Pompeu Fabra
- Universidad Pontificia Comillas

- Universidad Pontificia de Salamanca

- Universidad Ramón Llull

- Universidad Rey Juan Carlos

- Universidad Rovira i Virgili

- Universidad de San Jorge

- Universidad San Pablo-CEU

- Universidad de Santiago de Compostela

- Universidad de Sevilla

- Universidad de València (Estudi General)

- Universidad de Valladolid

- Universidad Vic-Central de Catalunya

- Universidad de Zaragoza

4. Curso en el que esté matriculado actualmente (la mayoría de las asignaturas)

- Primero

- Segundo

- Tercero

- Cuarto

5. Localidad donde ha pasado el confinamiento

(Respuesta corta)

6. ¿En qué grado le ha afectado la COVID-19?

- La COVID-19 no me ha afectado en ningún aspecto sanitario o económico

- La COVID-19 ha afectado a mi economía o a la de mi familia (ERTEs, cierre de negocios, autónomos, bajada de ingresos, etc.)

- La COVID-19 ha afectado a mi salud o a la de mi familia (contagiados o fallecidos por la enfermedad)

- La COVID-19 ha afectado a la salud (contagiados o fallecidos por la enfermedad) y a la economía propia o la de mi familia (ERTEs, cierre de negocios, autónomos, bajada de ingresos, etc.)

Segunda parte de la encuesta: preguntas sobre el consumo mediático durante el estado de alarma

7. ¿Por qué vía se enteró de que en España se decretó el estado de alarma?

- Por llamada telefónica

- Por mensajería instantánea (WhatsApp, Telegram, etc.)

- Por una alerta en su dispositivo electrónico

- Por redes sociales (comparecencia de Pedro Sánchez en directo) 
- Por mensajes publicados en redes sociales

- Por radio (comparecencia de Pedro Sánchez en directo)

- Por informativo/programa/podcast de radio

- Por televisión (comparecencia de Pedro Sánchez en directo)

- Por informativo/programa de televisión

- Por prensa digital (comparecencia de Pedro Sánchez en directo)

- Por prensa digital a través de piezas informativas

- Otras

- Ns /Nc

8. A raíz de que decretó el estado de alarma, tu consumo de información sobre la COVID-19

- Aumentó

- Disminuyó

- Se mantuvo igual

- Ns / Nc.

9. A lo largo del confinamiento...

- Mi consumo informativo no ha variado. Se ha mantenido constante.

- Mi consumo informativo ha ido descendiendo según han pasado las semanas.

- Mi consumo informativo ha ido aumentado según han pasado las semanas.

- Ns / Nc.

10. En general, señale con qué frecuencia ha consultado información sobre la COVID-19

- No he consultado información sobre la COVID-19 a diario.

- Una vez al día.

- Entre dos y cuatro veces al día.

- Cinco o más veces al día.

- Cada vez que recibo una alerta en mis dispositivos electrónicos.

- Ns / Nc.

11.1. Principalmente, ¿a través de qué vía se ha informado sobre la COVID-19?

- Periódicos impresos.

- Periódicos digitales.

- Radio.
- Redes sociales (Facebook, Twitter, Instagram, etc.).

- Servicios de mensajería (WhatsApp, Telegram, etc.).

- Informativos de televisión.

- Otros programas de televisión.

- Comparecencias institucionales.

- Boletín Oficial del Estado (BOE).

- Ns / Nc.

11.1.1. Especifique (dentro de la opción elegida) el medio / la empresa en concreto:

(Pregunta abierta)

11.2. ¿Y en segundo lugar?

- Periódicos impresos.

- Periódicos digitales.

- Radio.

- Redes sociales (Facebook, Twitter, Instagram, etc.).

- Servicios de mensajería (WhatsApp, Telegram, etc.).

- Informativos de televisión.

- Otros programas de televisión.

- Comparecencias institucionales.

- Boletín Oficial del Estado (BOE).

- Ns / Nc.

11.2.1. Especifique (dentro de la opción elegida) el medio / la empresa en concreto

(Pregunta abierta)

12. A raíz de la declaración del estado de alarma, ¿qué tipo de contenidos ha consumido más?

- Información.

- Interpretación (reportajes, entrevistas, etc.).

- Opinión.

- No ha aumentado mi consumo mediático.

- Ns / Nc.

13. Antes de la declaración del estado de alarma, ¿qué tipo de contenidos consumía más?

- Información.

- Interpretación (reportajes, entrevistas, etc.).

- Opinión.

- No ha aumentado mi consumo mediático.

- Ns / Nc. 
Tercera parte de la encuesta: preguntas sobre el tratamiento informativo de los medios respecto a la covid-19

14. Los medios de comunicación se han considerado en esta pandemia servicios fundamentales. Por tanto, ¿considera que, a raíz de esta situación, ha aumentado la credibilidad de los mass media?

$$
\begin{aligned}
& \text { - Sí } \\
& \text { - No } \\
& \text {-Ns / Nc }
\end{aligned}
$$

15. Señale la afirmación con la que está más de acuerdo en relación de la cobertura mediática de la COVID-19

- En general, los medios están ofreciendo poca información sobre la COVID-19

- En general, los medios están ofreciendo una información proporcional la relevancia social de la COVID-19

- En general, los medios están ofreciendo excesiva información sobre la COVID-19

- Ns / Nc

16. ¿Alguna vez creyó que una información sobre la COVID-19 era cierta y luego supo que era falsa?

- Sí

- No

-Ns / Nc

17. Sobre las informaciones acerca de la COVID-19 proporcionadas por los medios de comunicación:

- Considero que la mayoría de informaciones están perfectamente contrastadas y son veraces.

- Considero que la mayoría de informaciones carecen de una buena labor de contraste.

- Considero que la mayoría de informaciones están sesgadas ideológicamente.
- Considero que muchas de ellas contradicen informaciones anteriores y sólo generan confusión.

- Ns / Nc.

18.1. De estas cuestiones, ¿qué tipo de información deberían haber priorizado los medios en primer lugar durante la pandemia?

- Estadísticas diarias / información sanitaria.

- Información económica y política.

- Historias de superación personal.

- Iniciativas solidarias.

- Aprendizaje sobre el lavado de manos, uso de mascarillas, distancia de seguridad, etc.

- Información sobre las distintas fases de la «desescalada».

- Ns / Nc.

18.2. ¿Y en segundo lugar?

- Estadísticas diarias / información sanitaria.

- Información económica y política.

- Historias de superación personal.

- Iniciativas solidarias.

- Aprendizaje sobre el lavado de manos, uso de mascarillas, distancia de seguridad, etc.

- Información sobre las distintas fases de la «desescalada».

- Ns / Nc.

19.1. Durante el estado de alarma, ¿̇ha ejercido la labor periodística en algún medio de comunicación con vinculación laboral o como estudiante en prácticas?

- Sí.

- No.

19.1.1. Si es que sí, especifique cuál:

(Pregunta abierta) 